\title{
Topographic Core-Mantle Coupling and Fluctuations in the Earth's Rotation
}

\author{
R. HIDE \\ Robert Hooke Institute, The Observatory \\ Clarendon Laboratory, Parks Road \\ Oxford OXI 3PU, England, U. K. \\ R. W. CLAYTON \\ Seismological Laboratory, California Institute of Technology \\ Pasadena, CA 91125. U.S. A. \\ B. H. HAGER \\ Department of Earth, Atmospheric and Planetary Sciences \\ Massachusetts Institute of Technology \\ Cambridge, MA 02139, U.S.A. \\ M. A. SPIETH \\ Jet Propulsion Laboratory, California Institute of Technology \\ Pasadena, CA 91109, U.S. A. \\ C. V. VOORHIES \\ Geodynamics Branch Code 921, Goddard Space Flight Center \\ Greenbelt, MD 20771, U. S. A.
}

\begin{abstract}
Astronomically-determined irregular fluctuations in the Earth's rotation vector on decadal time scales can be used to estimate the fluctuating torque on the lower surface of the Earth's mantle produced by magnetohydrodynamic flow in the underlying liquid metallic core. A method has been proposed for testing the hypothesis that the torque is due primarily to fluctuating dynamic pressure forces acting on irregular topographic features of the core-mantle boundary and also on the equatorial bulge. The method exploits (a) geostrophically-constrained models of fluid motions in the upper reaches of the core based on geomagnetic secular variation data, and (b) patterns of the topography of the CMB based on the mantle flow models constrained by data from seismic tomography, determinations of long wavelength anomalies of the Earth's gravitational field and other geophysical and geodetic data. According to the present study, the magnitude of the axial component of the torque implied by determinations of irregular changes in the length of the day is compatible with models of the Earth's deep interior characterized by the presence of irregular CMB topography of effective "height" no more than about $0.5 \mathrm{~km}$ (about $6 \%$ of the equatorial bulge) and strong horizontal variations in the properties of the D" layer at the base of the mantle. The investigation is now being extended to cover a wider range of epochs and also the case of polar motion on decadal time scales produced by fluctuations in the equatorial components of the torque.
\end{abstract}

\section{INTRODUCTION}

Electric currents generated in the Earth's liquid metallic core are responsible for the main geomagnetic field and its secular changes [see Jacobs, 1987ab; Melchior, 1986; Moffatt, 1978a]. The currents

Relating Geophysical Structures and Processes: The Jeffreys Volume Geophysical Monograph 76, IUGG Volume 16

Copyright 1993 by the International Union of Geodesy and Geophysics and the American Geophysical Union. are produced by dynamo action involving irregular magnetohydrodynamic flow in the core. Concomitant dynamical stresses acting on the overlying mantle are invoked in the interpretation of the so-called "decadal" fluctuations in the rotation of the "solid Earth" (mantle, crust and cryosphere). Studies of these rotational manifestations of core motions bear directly on investigations of the structure, composition and dynamics of the Earth's deep interior [Aldridge, 1990; Anufriev and Braginsky, 1977; Benton, 1979; 
Eltayeb and Hassan, 1979; Hide, 1969; 1970; 1977; 1986; 1989; Hide and Dickey, 1991; Hinderer et al., 1990; Jault and Le Mouël, 1989; 1990; Lambeck, 1980; 1988; Moffatt, 1977b; Morrison, 1979; Paulus and Stix, 1989; Roberts, 1972; Rochester, 1984; Spieth et al., 1986; Voorhies, 1991a; Wahr, 1988].

Consider a set of body-fixed axes $x_{i}, i=1,2,3$, aligned with the principal axes of the solid Earth and rotating about the center of mass of the whole Earth with angular velocity

$$
\hat{\omega}_{i}=\hat{\omega}_{i}(t)=\left(\hat{\omega}_{1}, \hat{\omega}_{2}, \hat{\omega}_{3}\right)=\Omega\left(\hat{m}_{1}, \hat{m}_{2}, 1+\hat{m}_{3}\right)
$$

Here $t$ denotes time and $\Omega$ the mean speed of rotation of the solid Earth in recent times, $0.7292115 \times 10^{4}$ radians per second [Cazenave, 1986; Lambeck, 1980; Moritz and Mueller, 1987; Munk and MacDonald, 1960; Rochester, 1984]. Over time scales short compared with those characteristic of geological processes, the rotation of the Earth departs only slightly from steady rotation about the polar axis of figure, so that $\hat{m}_{1}, \hat{m}_{2}$, and $\hat{m}_{3}$ are all very much less than unity and $\left|m_{i}\right| \ll \Omega$, where $\dot{\hat{m}}_{i} \equiv d \hat{m}_{i} / d t$. Periodic variations in $\hat{\omega}_{i}$ on time scales less than a few years are caused by periodic lunar and solar tidal torques and related changes in the moment of inertia of the solid Earth. Irregular variations on these time scales are produced by atmospheric and oceanic torques due to tangential stresses in surface boundary layers and normal pressure forces acting on surface topography, and they are largely associated with seasonal, intraseasonal and interannual fluctuations in the total angular momentum of the atmosphere. When these rapid variations (including the Chandlerian wobble of the figure axis relative to the rotation axis) have been removed from the observational data, the smoothed time series

$$
\omega_{1}=\Omega\left(m_{1}, m_{2}, 1+m_{3}\right)
$$

that remains reveals (within the errors involved) the decadal variations, the axial component of which is illustrated in Fig. 1. Geophysicists have long argued that these decadal variations in $m_{3}$ are largely manifestations of angular momentum exchange between the core and mantle [Jacobs, 1987ab; Jault and Le Mouël, 1991; Stoyko, 1951; Vestine, 1952].

Expressions needed in the study of the variable rotation of the non-rigid solid Earth due to core-mantle coupling are readily obtained by standard methods based on Euler's dynamical equations (see e. g., Munk and MacDonald, [1960]). If $L_{i}^{*}(t), i=1,2,3$, is the fluctuating torque exerted by the core on the mantle then the axial component $L_{3}^{*}$ satisfies [Hide, 1989]:

$$
\dot{\mathrm{m}}_{3}=-\Lambda_{0} \mathrm{~d}(\Delta \Lambda) / \mathrm{dt}=\mathrm{L}_{3}{ }^{*} / \Omega \mathrm{C}+\alpha_{3}
$$

where $C$ is the principal moment of inertia of the solid Earth about the polar axis, $\Lambda_{0} \equiv 2 \pi / \Omega$ is the average length of the day (LOD), $\Lambda(t) \equiv 2 \pi / \omega_{3}$ and $\Delta \Lambda \equiv \Lambda(t)-\Lambda_{0}$. The quantity $\alpha_{3}$, which can be calculated explicitly [Hide, 1989], represents secondary effects due to a variety of causes, such as fluctuations in the inertia tensor of the solid Earth (including changes associated with stresses responsible for $L_{i}^{*}$ and to comparatively weak torques applied at the Earth's surface by the atmosphere and oceans on the relevant time scales).
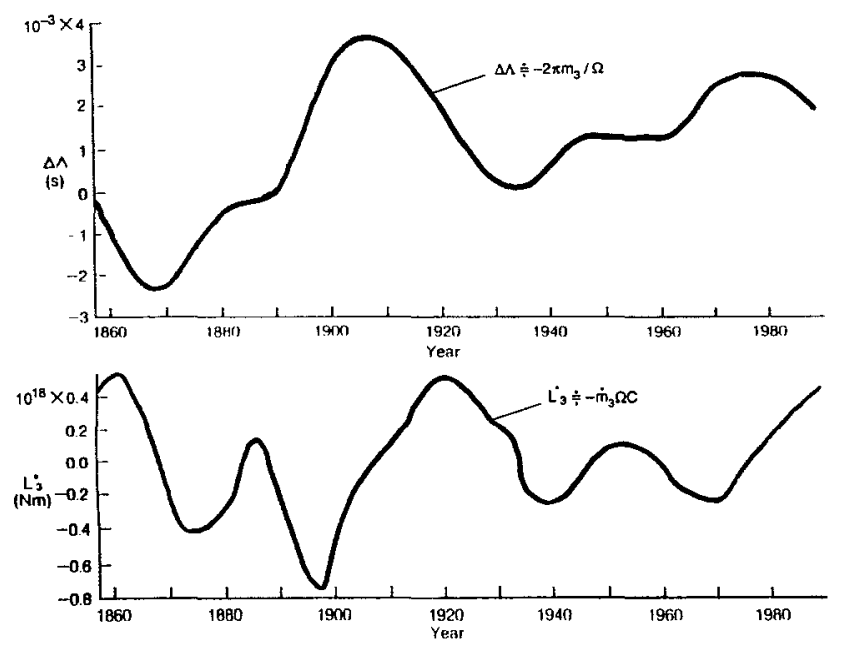

Fig. 1. Decadal variations in the length of day $\Lambda_{0}+\Delta \Lambda(t)$ from 1855 to 1985 (upper curve) and corresponding variations in the axial component $L 3^{*}(t)$ of the torque $L^{*}$ acting upon the solid Earth (lower diagram); see equation (1.3) and Dickey et al. [1989] or Hide and Dickey [1991]. More rapid variations (of tidal and atmospheric origin) were removed from the original time series by taking a ten-year running average.

\section{THE TORQUE EXERTED By THE CORE ON THE MANTLE}

The fluctuating torque $L_{i}^{*}(t)$ exerted by the core on the overlying mantle is largely a consequence of (a) tangential stresses at the CMB associated with shearing motions in the thin (less than $1 \mathrm{~m}$ ) viscous boundary layer just below the $\mathrm{CMB}$, (b) normal dynamical pressure forces acting on the equatorial bulge and other (possibility) smaller and more irregular departures from spherical of the shape of the CMB, (c) Lorentz forces due to the flow of electric currents in the weakly-conducting lower mantle generated by the electromotive forces associated with the geodynamo processes in the core and (d) gravitational forces associated with horizontal density variations in the core and mantle and especially at the CMB [see Hide, 1969; Jacobs, 1987ab; Jault and Le Mouël, 1991; Melchoir, 1986; Rochester, 1984; Voorhies, 1991a]. Denote by $L_{i(+)}^{*}$ and $L_{i}^{*}(-)$ the respective contributions to $L_{i}^{*}$ from regions where tractions act in the positive sense and from regions where tractions act in the negative sense and introduce the "canceling factor"

$$
\gamma_{i}^{*}=\gamma_{i}^{*}(t) \equiv L_{i}^{*} /\left[L_{i(+)}^{*}-L_{i(-)}^{*}\right]
$$

By definition, each $\gamma_{i}$ fluctuates about an average of zero and attains magnitudes less than or equal to unity. The accuracy with which $L_{i}^{*}$ can be determined obviously depends on the value of $\left|\gamma_{i}^{*}\right|$, the most favorable situation being when $\left|\gamma_{i}^{*}\right| \sim 1$.

Rough dynamical arguments show that the pressure coupling associated with CMB topography might predominate over other effects [Hide, 1969]. The contribution made by viscous stresses is negligible on all but the most extreme assumptions about the viscosity of the core, but electromagnetic coupling, according to detailed studies by a number of investigators, might be adequate if the (unknown) electrical conductivity of the lower mantle were suffi- 
ciently high and the toroidal magnetic field in the core concentrated in a boundary layer just below the CMB [Bullard et al., 1950; Paulus and Stix, 1989; Roberts, 1972; Rochester, 1984]. So it is of interest to investigate the topographic contribution $L_{i}(t)$ (say) to $L_{i}^{*}(t)$ using available geophysical data, in order to establish the extent to which decadal variations in the Earth's rotation might reasonably be attributed to topographic core-mantle coupling.

If $p_{s}$ is the dynamical pressure associated with core motions $\boldsymbol{u}=u_{s}$ in the free stream just below the viscous boundary layer at the $\mathrm{CMB}$ ( $\boldsymbol{u}$ being the Eulerian flow velocity relative to a reference frame fixed to the solid Earth) and the CMB is the locus of points where the distance from the Earth's center of mass is $r=c+h(\theta, \phi)$, ( $c$ being the mean radius of the CMB and $(\theta, \phi)$ the co-latitude and longitude of a general point), then [see Hide, 1989; Voorhies, $1991 a]$.

$$
L_{i}(t)=-c^{2} \int_{0}^{2 \pi} \int_{0}^{\pi}\left(r \times p_{s} \nabla h\right)_{i} \sin \theta d \theta d \phi
$$

if $|h|<<c$ and $(\nabla, h)<<1$. Here $\nabla_{s} \equiv c^{-1}(\hat{\theta} \partial / \partial \theta, \hat{\phi} \operatorname{cosec} \theta \partial / \partial \phi)$, $\hat{\theta}$ and $\hat{\phi}$ are unit vectors in the directions of increasing $\theta$ and $\phi$ respectively, and $\boldsymbol{r}$ is the vector distance from the Earth's center of mass. An important step in the analysis is the recognition of the fact that the surface integral of $r \times \nabla_{s}\left(h p_{s}\right)$ over the whole CMB is equal to zero, which leads to a more useful expression for $L_{i}(t)$, namely

$$
L_{i}(t)=c^{2} \int_{0}^{2 \pi} \int_{0}^{\pi}\left(r \times h \nabla p_{s}\right)_{i} \sin \theta d \theta d \phi
$$

Nearly everywhere within the core, owing to the presence there of electric currents, Lorentz forces may be comparable in magnitude with the Coriolis forces due to the Earth's rotation acting on core flow. But in the upper reaches of the core, within tens of kilometers of the CMB, Lorentz forces should be about $10^{-2}$ times the Coriolis forces or less, unless geodynamo action is confined to a thin boundary layer just below the CMB or metallic electrical conductivities are attained at the base of the "solid" mantle. In regions where Lorentz forces are negligible, geostrophic balance between the horizontal components of the pressure gradient and Coriolis forces should obtain to sufficient accuracy [Backus and Le Mouël, 1986; 1987; Bloxham and Jackson, 1991; Gire and Le Mouel, 1990; Hide, 1986; 1989; Hills, 1979; Le Mouël, 1984; Voorhies, 1991a]. Whence

$$
2 \bar{p}_{s} \Omega \cos \theta\left(-\mathrm{w}_{\mathrm{s}}, \mathrm{v}_{\mathrm{s}}\right)=-\mathrm{c}^{-1}\left(\partial \mathrm{p}_{\Omega} / \partial \theta, \operatorname{cosec} \theta \partial \mathrm{p}_{\mathrm{J}} / \partial \phi\right)
$$

where $\bar{\rho}_{s}$ is the horizontally averaged value of the density $\rho$ in the upper-reaches of the core. Here $\left(v_{s}, w_{s}\right)$ are the $(\theta, \phi)$ components of $\boldsymbol{u}_{\boldsymbol{*}}$, which are typically much greater in magnitude than $u_{r}$, the $r$ component of $\boldsymbol{u}_{\boldsymbol{z}}$. It follows from equations (2.3) and (2.4) that on the time scales of interest here, over which $u_{z}$ may change significantly but $h$ does not, that

$$
L_{3}(t)=-2 \bar{\rho}_{s} \Omega c^{3} \int_{0}^{2 \pi} \int_{0}^{\pi} h(\theta, \phi) \nu_{s}(\theta, \phi, t) \sin ^{2} \theta \cos \theta d \theta d \phi
$$

The basic theoretical relationships needed are given by equation (1.3) with $\alpha_{3}=0$ and the working hypothesis that $L_{3}^{*}=L_{3}$, together with equations (2.2) to (2.5). The integral on the right-hand side of equation (2.5) involves CMB topography $h(\theta, \phi)$. When dealing with the equatorial components of the torque and the polar motion they produce, the dominant contribution to $h$ is the equatorial $\phi$ independent bulge of the CMB, which corresponds to a $9 \mathrm{~km}$ difference between the equatorial and polar radii of the core. [e.g., Herring et al., 1991]. But the equatorial bulge makes no contribution to the axial component $L_{3}$, which changes the LOD $\Lambda(t)$ (see equation (1.3)), so when dealing with such changes it is necessary to look in detail at features of $h$ that depend on $\phi$ as well as $\theta$. Over the past-twenty years various attempts have been made to infer $h(\theta$, $\phi)$ from seismology and from the pattem of long-wavelength gravity anomalies under various assumptions about the structure and rheology of the solid Earth, with more recent studies making use of results from seismic tomography; several hypothetical fields of $h(\theta$, $\phi)$ are now available, as discussed in Section 4 below.

The other quantity needed in the evaluation of $L_{i}(t)$ is either the field of pressure $p_{s}(\theta, \phi, t)$ or the field of horizontal flow $\left(\nu_{s}(\theta, \phi, t)\right.$, $\left.w_{s}(\theta, \phi, t)\right)$, which is related to $p_{s}$ through equation (2.4). Geomagnetic secular variation data have been used by various workers to infer $\left(v_{s}, w_{s}\right)$ by a method that invokes the geostrophic relationship (equation (2.4)) in combination with the equations of electrodynamics appropriate to the case when the mantle can be treated as a perfect electrical insulator of uniform magnetic permeability and the core as a perfect conductor, as we shall now discuss.

\section{Velocity and PRESSURE FIELdS IN THE CORE}

Denote by $\boldsymbol{B}(r, \theta, \phi, t)$ the value of the main geomagnetic field at a general point $(r, \theta, \phi)$ at time $t$, and by $\dot{B} \equiv \partial B / \partial t$ the so-called geomagnetic secular variation. Determinations of $\boldsymbol{B}$ made at and near the Earth's surface at various epochs can be used to infer $u_{n}$, the Eulerian flow velocity just below the CMB [Backus and Le Mouël, 1986; 1987; Benton, 1981ab; Benton and Celaya, 1991; Bloxham, 1988; 1989; Bloxham and Jackson, 1991; Courtillot and Le Mouël, 1988; Gire et al., 1986; Gire and Le Mouel 1990; Gubbins, 1982; Lloyd and Gubbins, 1990; Voorhies, 1986ab; 1987; 1991; 1992; Voorhies and Backus, 1985; Whaler, 1986; 1990; 1991; Whaler and Clarke, 1988]. From flows that are constrained to be tangentially geostrophic (see below), an estimate of the horizontal pressure gradient just below the CMB can be deduced through equations (2.4).

The first of the three reasonable key assumptions that underlie the method used is that the electrical conductivity of the mantle and magnetic permeability gradients there are negligibly small, so that $\boldsymbol{B}$ can be written as the gradient of a potential $V$ satisfying Laplace's equation $\nabla^{2} V=0$. This facilitates the downward extrapolation of the observed field at and near the Earth's surface in order to obtain $B$ and $\dot{\boldsymbol{B}}$ at the CMB.

The second assumption is that the electrical conductivity of the core is so high that when dealing with fluctuations in $B$ on time scales very much less than that of the Ohmic decay of magnetic fields in the core (which is several thousand years for global-scale features), $\boldsymbol{B}$ satisfies Alfven's "frozen flux" theorem [Backus, 1968; Roberts and Scott, 1965]. This is expressed by the equation

$$
\partial \mathrm{B} / \partial \mathrm{t}=\nabla \times(\mathbf{u} \times \mathbf{B}),
$$

which may be shown to imply that the lines of magnetic force 
emerging from the core are advected by the horizontal flow $\left(v_{s}, w_{s}\right)$ just below the CMB. Accordingly, if $B=\left(B_{r}, B_{\theta}, B_{\phi}\right)$, then $B_{r}$ at the CMB satisfies

$$
\frac{\partial B_{r}}{\partial t}+\frac{\nu_{s}}{c} \frac{\partial B_{r}}{\partial \theta}+\frac{w_{s}}{c \sin \theta} \frac{\partial B_{r}}{\partial \phi}=B_{r}\left[\frac{\partial u}{\partial r}\right]_{r=c}
$$

Now equation (3.1) alone does not permit the unique determination

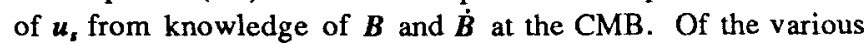
additional considerations that have been employed to secure uniqueness, a physically plausible approach is to invoke the assumption we have already made in Section 2, namely that the flow in the upper reaches of the core is in geostrophic balance with the pressure field there [Backus and Le Mouell, 1986; 1987; Hills, 1979; Jault et al., 1988; Le Mouël, 1984]. The corresponding radial vorticity balance can be expressed by the equation

$$
\cos \theta\left[\frac{\partial u}{\partial r}\right]_{r=c}+\frac{\nu_{s}}{c} \sin \theta=0
$$

which is readily deduced by eliminating $p_{s}$ from equations (2.4) and using the mass continuity equation $\nabla \cdot u=0$ for flow in an effectively incompressible fluid.

Various groups of geomagnetic workers have produced maps of $\left(v_{s}, w_{s}\right)$ and investigated the errors and uncertainties encountered in practice [Bloxham and Jackson, 1991]. In this study, we use ten flow models, two of which have been published elsewhere and eight of which were produced for this study. Four of these models are shown in Figure 2. Figure 2a shows model IIa of Bloxham [1989], a geostrophic model determined assuming steady flow and using a smoothly varying model of the magnetic field at the CMB for the interval 1975 - 1980. This model is expressed in terms of spherical harmonics through degree and order 14 . The model shown in Figure 2b is model GVC1E6 of Voorhies [1991a; 1992], derived, assuming steady flow, using $B$ and $\dot{B}$ from the "Definitive Geomagnetic Reference Field" (DGRF) at epochs starting in 1980 and moving back to 1945 in 5-year intervals. This model incorporates spherical harmonic expansions up to degree and order 16 and provides a weighted variance reduction of $98.074 \%$. Voorhies [1988] presented a series of models fit to the DGRF for shorter intervals of time. Model G6070.1, shown in Figure 2c, is a relatively smooth fit to the DGRF from 1960 - 1970. Model G8070.1, shown in Figure 2d, uses the same damping parameter to fit the DGRF data from 1980 - 1970. Other models of the $\mathrm{G} 6070 . \mathrm{m} / \mathrm{G} 8070 . \mathrm{m}$ series (not shown here) for the same time intervals were used, with increasing index $m$ corresponding to increasing roughness of the model flows.

These flow models, which were used to produce hypothetical flow fields $u_{x}$ and corresponding pressure fields $p_{s}$, are all similar in appearance. Voorhies' [1988; 1991a; 1992] models CVC1E6 and G8070.1 are barely distinguishable in these plots, reflecting the fact that the models were fit using a similar procedure starting with the 1980 DGRF as the initial condition. The most apparent differences between these two models and the model of Bloxham [1989], appropriate for approximately the same time span as G8070.1, are beneath southern Africa and in the northwestern Pacific. The differences between Voorhies' [1988] models G8070.1 and G6070.1, fit to different epochs of the DGRF using identical procedures, are also small, being most easily seen beneath northern Siberia, Alaska, and the southeastern Pacific. Although the calculation of the time-varying core surface flows needed to model variations in $p_{s}$, (hence $L_{i}$ ) is straightforward, the similarity among these models demonstrates the care needed to estimate such variations reliably. In particular, there is as much variation between models fit to the same epoch by different workers as there is between models by a single worker of the flow for different epochs.

\section{TOPOGRAPHY OF THE CORE-MANTLE BOUNDARY}

The topography $h(\theta, \phi)$ of the CMB (see equation (2.3)) has been investigated using various techniques. The problem is complicated

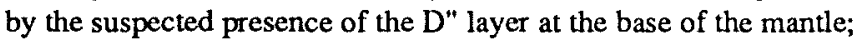
its variable mechanical and chemical properties make it difficult to separate the effects of $D^{\prime \prime}$ and CMB topography [Anderson, 1989; Bullen, 1963; Doornbos, 1980; Gubbins, 1989; Gudmundsson et al., 1990; Gudmundsson and Clayton, 1991, 1992; Haddon, 1982; Hager et al., 1985; Hager and Richards, 1989; Jacobs, 1987a; Jeanloz, 1990; Knittle and Jeanloz, 1991; Loper, 1991; Morelli and Dziewonski, 1987]. Determinations of the amplitude and phase of the free-core nutation lead to an estimate of $0.5 \mathrm{~km}$ of excess ellipticity at the CMB [Babcock and Wilkins, 1988; Gwinn et al., 1986; Herring et al., 1985; 1986; 1991; Kinoshita and Suchay, 1990; Reid and Moran, 1988; Wahr, 1988]. While this topographic component does not affect $L_{3}$ (and hence the LOD, see equation (1.3)), it may indicate the likely magnitude of the relief.

The most direct method (in principle) of estimating CMB topography is to examine variations in travel times of seismic waves that interact with the CMB. Core reflected phases such as PcP and phases that propagate through the core such as PKP and PKIKP are affected similarly by the presence of velocity anomalies in the mantle, which, for rays traversing the same path, affect the travel-times of both phases comparably. However, deformations of the CMB affect the two phases in opposite senses; in regions where $h<0$, $\mathrm{PcP}$ travel times are increased owing to increased path length, but PKP and PKIKP travel times are reduced because a greater portion of the path is through the "faster" mantle material. Potentially, a joint investigation of data from both types of phase could lead to a unique determination of seismic properties of the CMB [Morelli and Dziewonski, 1987]. Unfortunately, the quality of the observations of PcP, PKP, and PKIKP travel times given in the International Seismic Centre (ISC) catalogue tends to be poor, both in terms of travel-time measurements and geographic distribution.

Morelli and Dziewonski [1987] boldly carried out inversions for CMB topography using PcP and PKPef phases. The models for these two phases showed a reasonable correlation, which they took as evidence that the inversions were successful, despite the potential pitfalls. They inverted both data sets together to determine a model of CMB topography through degree and order 4, with peak-to-peak amplitudes in excess of $10 \mathrm{~km}$.

Gudmundsson and Clayton [1992] carried out a number of inversions of the ISC data for these phases, as well as the additional phases PKPab, PKPbc; and PKPde. They also investigated the 
(a) $\begin{array}{lllllllllllll}0 & 30 & 60 & 90 & 120 & 150 & 180 & 210 & 240 & 270 & 300 & 330 & 360\end{array}$

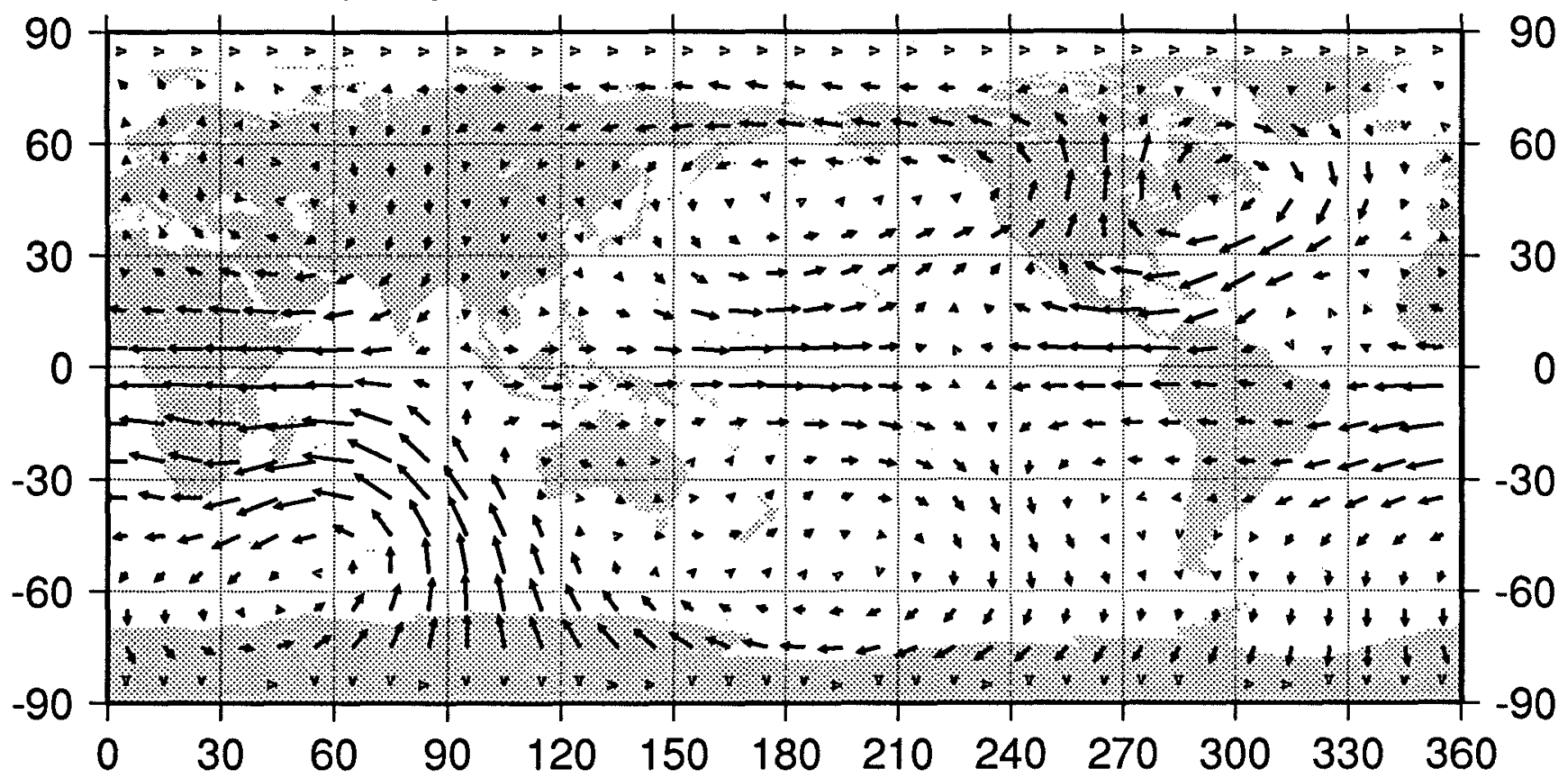

$\begin{array}{lllllllllllll}\text { (b) } 0 & 30 & 60 & 90 & 120 & 150 & 180 & 210 & 240 & 270 & 300 & 330 & 360\end{array}$

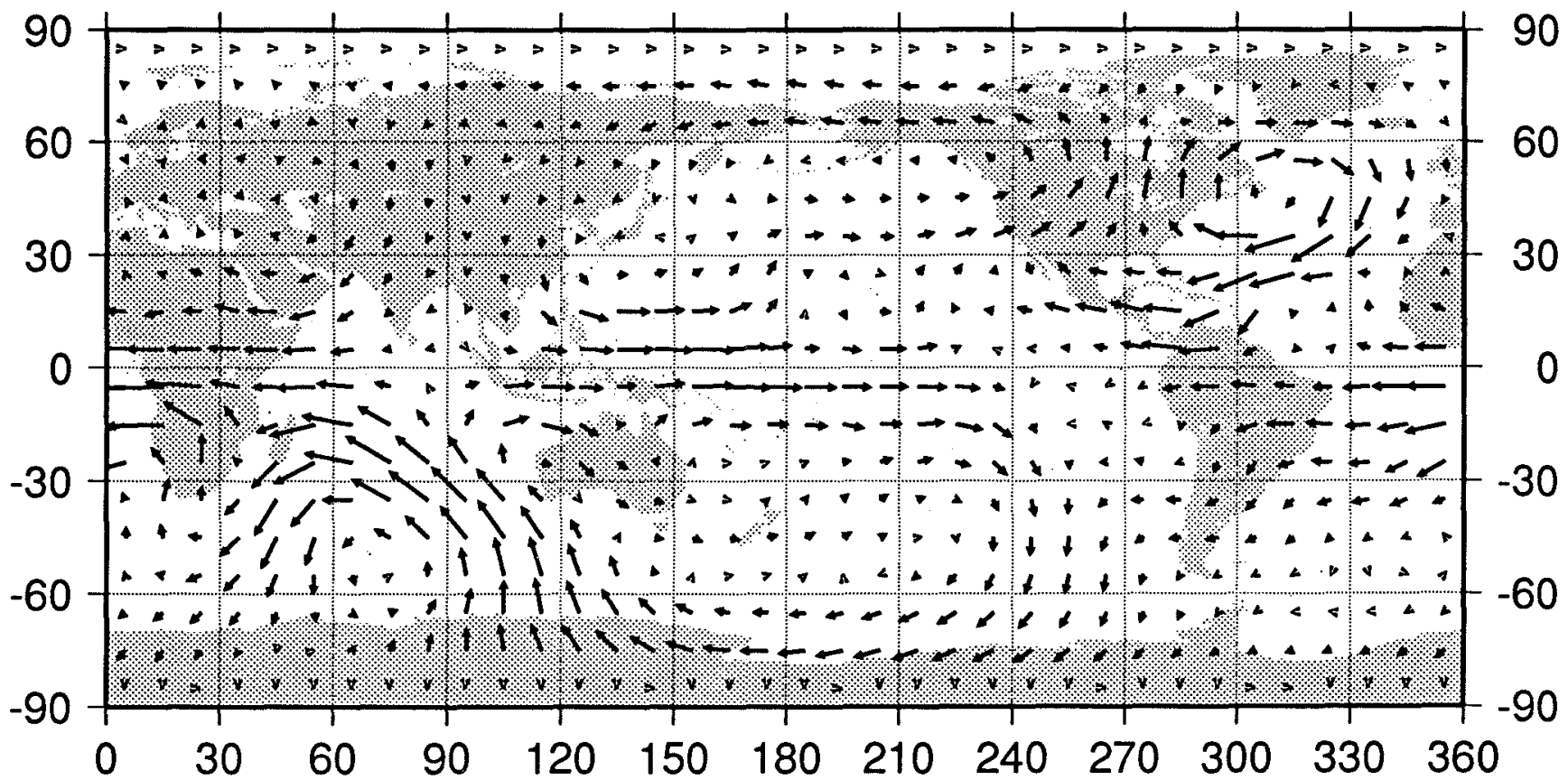

Fig. 2. Geostrophic flow fields estimated by Bloxham [1989] (2a), Voorhies [1991a] (2b) and Voorhies [1988] (2c and $2 \mathrm{~d}$ ). An arrow with a magnitude equal to the distance between grid points $\left(10^{\circ}\right)$ corresponds to a velocity of 20 $\mathrm{km} / \mathrm{year}$. 
(c)

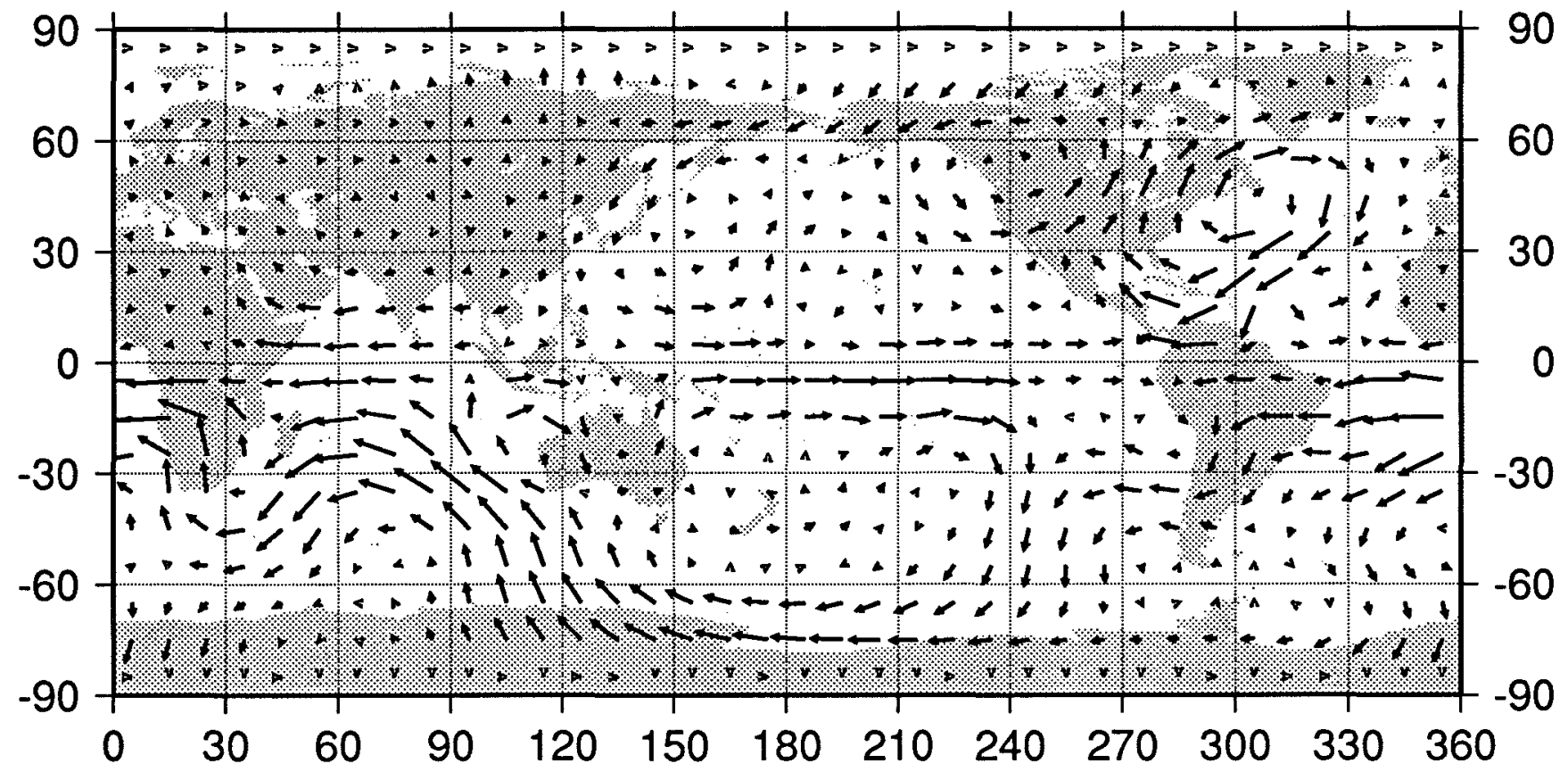

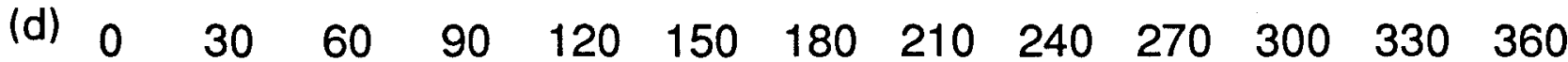

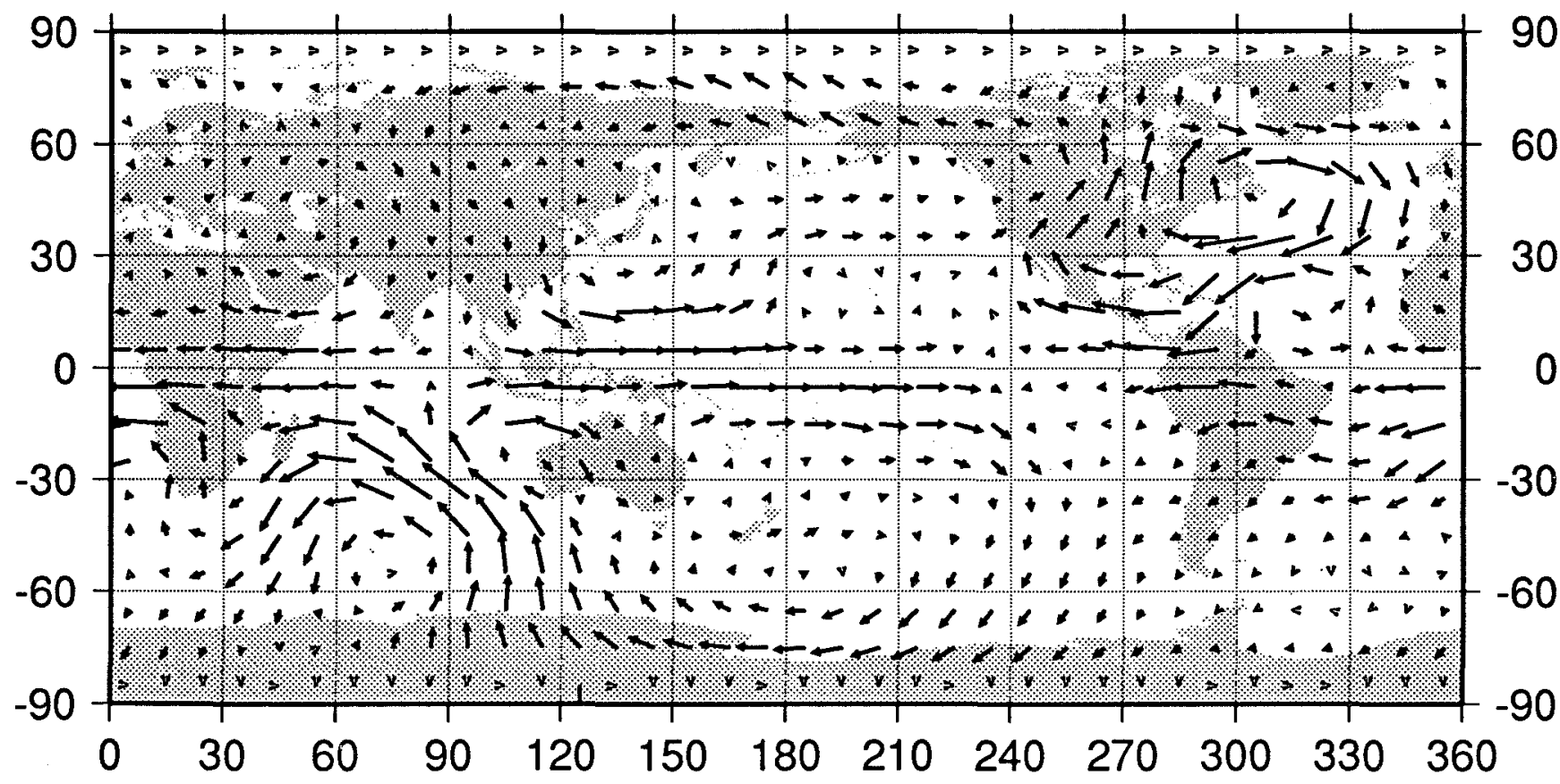

Fig. 2. (continued) 
effects of including a horizontally-variable D" layer in their models. The CMB topography obtained for the different phases differs substantially. In addition, they found that by varying the "tradeoff" between the effects due to $D$ " variations and CMB topography respectively, the amplitude of the $\phi$-dependent part of $h$ ranged from $1 \mathrm{~km}$ to $6 \mathrm{~km}$. Their models were parameterized in terms of spherical harmonic expansions through degree and order 10, although they employed a "stochastic inverse," choosing the trade-off parameter to retain only 25 parameters (as measured by the trace of the resolution matrix) in their solution. The topography for one particular choice that includes the effects of variable velocity in the D" layer in the model parameterization (Figure 9 in Gudmundsson and Clayton [1992]), expanded through degree and order 4 for comparison with the model of Morelli and Dziewonski [1987], is illustrated in the top panel of Figure 3.

Another approach to modeling CMB topography is to use density variations inferred from lateral variations in seismic-wave velocities in the mantle or other geophysical inference to drive models of mantle flow. These models, which predict dynamically-maintained topography at the Earth's surface and at the CMB, are constrained by long-wavelength features of the geoid. This approach assumes that the seismic-velocity variations are caused by density variations (via temperature), to which they are linearly related. The mantle viscosity must also be specified, along with major chemical boundaries in the mantle, including the problematic $D^{\prime \prime}$ layer. In a spherical harmonic expansion of $h(\theta, \phi)$ up to degree and order 9 , models with no D" layer give amplitudes of the $\phi$-dependent part of $h$ up to $2.5 \mathrm{~km}$ (e.g., model WO of Hager and Clayton [1989]), nearly $25 \%$ of the equatorial bulge. On the other hand, models with a low viscosity and/or chemically distinct D" layer give much lower amplitudes. For example, the CMB topography for model WL of Hager and Richards [1989], a model which has a D" layer which has a low viscosity, but is not compositionally distinct from the overlying mantle, is shown in the bottom panel of Figure 3. It has an excess ellipticity compatible with the inferences from nutation studies. The model is dominated by long-wavelength variations, with a peak-topeak amplitude of the topography of $<2 \mathrm{~km}$.

Clearly, models of CMB topography differ more than do models of core flow. Thus, the comparison of the implied LOD variations with observed values on the basis of the method outlined in Section 1 has the potential to distinguish among classes of models such as those presented here.

\section{PREDICTED TORQUES AND LENGTH-OF-DAY VARIATIONS}

Estimates of the topographic torque exerted by the core on the mantle were produced from models of the flow fields in the outer core and the CMB topography via numerical integration of equation (2.5), and compared with values implied by the LOD determinations presented in Figure 1. Predicted LOD values obtained on the basis of equations (1.3) and (2.5) (with $\alpha_{3}=0$ and $L_{3}{ }^{*}=L_{3}$ ) for 50 combinations of 10 different models of the flow field, $\left(v_{s}, w_{s}\right)$, and 5 models of CMB topography, $h$, are given in Table 1 . Four of the core flow models are those shown in Figure 2; the others represent additional models presented by Voorhies [1988] for the DGRF in the epochs 1980-1970 and 1960-1970. The models differ in the damping parameter used to control the roughness of the flow. For example, G6070.1 is the smoothest model for 1960 - 1970, while G6070.4 is the roughest. In addition to the two CMB topography models shown in Figure 3, we also used the model of Morelli and Dziewonski [1987], model WO of Hager and Clayton [1989], and the model of Gudmundsson and Clayton [1992] shown in Figure 3, but expanded through degree and order 10.

Core flow Model IIa of Bloxham [1989] assumes steady flow during the epoch 1975-1980, during which time the average value of $L_{3}{ }^{*}$ was $-0.1 \times 10^{18} \mathrm{Nm}$; the corresponding change in LOD is about $-0.5 \mathrm{msec} / \mathrm{dec}$ ade. Models G8070.m of Voorhies [1988] assume steady flow starting in 1980 , going back to 1970 . During this interval, $\Delta \mathbf{\Lambda}$ first increased, then decreased, with negligible net torque averaged over the decade. Models G6070.m of Voorhies [1988] assume steady flow between 1960 and 1970, corresponding to $L_{3}{ }^{*}$ of $\sim-0.2 \times 10^{18} \mathrm{Nm}$ and a change in LOD of about +1 msec/decade. Model GVC1E6 of Voorhies [1991a] assumes steady flow starting in 1980, going back to 1945 , with the fit to the data being better at later than at earlier times in this interval. An average torque of $\sim-0.1 \times 10^{18}$ seems appropriate for the interval over which this model best fits the geomagnetic data; this would correspond to a change in LOD of $0.5 \mathrm{msec} / \mathrm{dec}$ ade.

By chance, all combinations of flow models and topography models presented here give positive predictions for changes in LOD. (Other models we have used, not shown, give negative predictions.) The combinations using the seismological models of $h$, with irregular $\phi$-dependent amplitudes of several kilometers, "predict" LOD changes much larger, some by over a factor of 100 , than the observed changes. Much closer agreements with the magnitude of observed LOD changes were found in the case of combinations for model WL, which has irregular CMB topography $<1 \mathrm{~km}$ in amplitude, although the predicted changes are still a factor of $4-8$ to large. For the topography models used here, Bloxham's [1989] flow model predicts the wrong sign of LOD variation during 1975 1980. Voorhies' [1988] models predict the correct sign for the decade represented by models G6070.m. The models for the epoch represented by models G8070. $m$ predict too large changes in LOD; the observed value for this decade is about zero. But, as discussed next, we are more confident of the order of magnitude of the torques than their sign, so the differences for these different flow models may not be diagnostic.

There is of course concern about the effect of inadequacies in the flow and topography fields on "predicted" values of the LOD variations. The problem is illustrated in Figure 4, which presents maps of the spatial distribution of contributions to the $L_{3}$ torque, $2 \bar{\rho}_{\mathrm{s}} \Omega_{0} c \sin \theta \cos \theta$, for the two flow models shown in Figures $3 \mathrm{c}$ and $3 \mathrm{~d}$, interacting with the topography shown in the bottom panel of Figure 2. As expected, a high degree of canceling is exhibited (see equation (2.1)). The net axial torque (and hence the LOD estimate) is the sum of these individual parts, which means it ends up being the small difference between two large numbers. For example, the total torque for the model in the top panel of Figure 4 is $-1 \mathrm{x}$ $10^{18} \mathrm{~N}$-m (comparable to the that inferred for $\sim 1900$, as can be seen from Figure 1). A constant torque per unit area of $-5 \times 10^{3} \mathrm{~N} / \mathrm{m}$ 

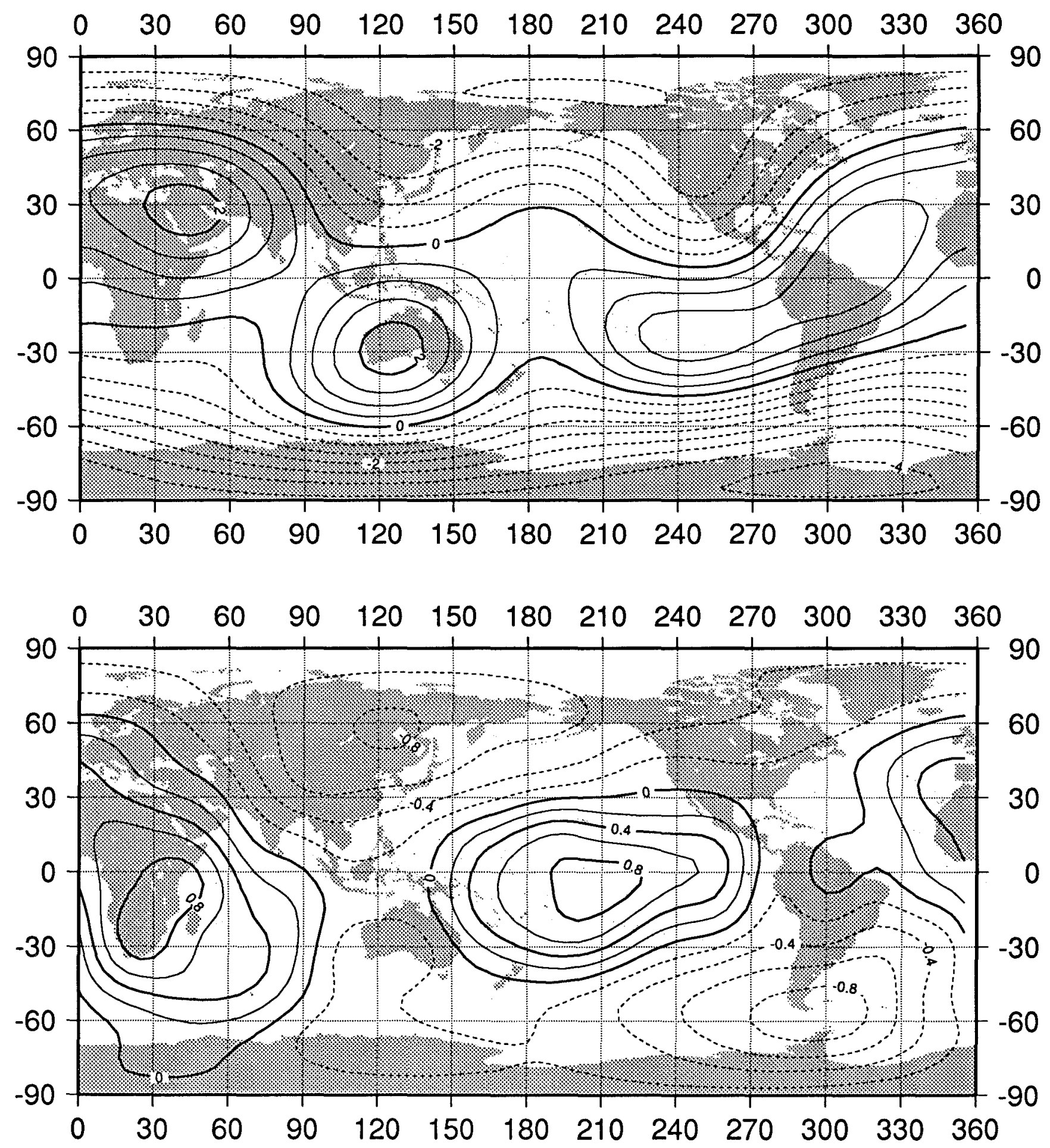

Fig. 3. Models of the topography of the CMB. The upper panel is a model derived using seismic tomography incorporating the effects of velocity variations in the D" layer above the CMB [Gudmundsson and Clayton, 1992]. The lower panel shows the dynamic topography inferred for model WL of Hager and Richards [1989]. Solid contours correspond to regions where the topography is upwarped towards the Earth's surface, while dashed contours represent regions where mantle is depressed into the core. The contour interval in the upper panel is $500 \mathrm{~m}$; in the lower panel, $200 \mathrm{~m}$. 
TABLE 1. Predicted values of LOD variations for combinations of CMB topography models and geostrophic flow models discussed in the text.

$\Delta \Lambda$ (msec/decade)

\begin{tabular}{|c|c|c|c|c|c|c|c|c|c|c|}
\hline $\begin{array}{c}\text { CMB } \\
\text { Topography } \\
\text { Flow } \\
\end{array}$ & $\begin{array}{c}\text { Bloxham } \\
\text { IIa } \\
{[1989]} \\
\end{array}$ & $\begin{array}{c}\text { Voorhies } \\
\text { GVC1E6 } \\
\text { [1992] }\end{array}$ & $\begin{array}{c}\text { Voorhies } \\
\text { G6070.1 } \\
{[1988]} \\
\end{array}$ & $\begin{array}{c}\text { Voorhies } \\
\text { G8070.1 } \\
{[1988]} \\
\end{array}$ & $\begin{array}{c}\text { Voorhies } \\
\text { G6070.2 } \\
\text { [1988] } \\
\end{array}$ & $\begin{array}{c}\text { Voorhies } \\
\text { G8070.2 } \\
\text { [1988] } \\
\end{array}$ & $\begin{array}{c}\text { Voorhies } \\
\text { G6070.3 } \\
{[1988]} \\
\end{array}$ & $\begin{array}{c}\text { Voorhies } \\
\text { G8070.3 } \\
{[1988]} \\
\end{array}$ & $\begin{array}{l}\text { Voorhies } \\
\text { G6070.4 } \\
{[1988]}\end{array}$ & $\begin{array}{c}\text { Voorhies } \\
\text { G8070.4 } \\
{[1988]} \\
\end{array}$ \\
\hline $\begin{array}{c}\text { Morelli \& } \\
\text { Dziewonski } \\
\text { [1987] }\end{array}$ & 143 & 115 & 110 & 133 & 115 & 131 & 97 & 120 & 58 & 112 \\
\hline $\begin{array}{c}\text { Gudmundsson } \\
\text { Clayton [1992] } \\
\text { degree 1-4 } \\
\end{array}$ & 85 & 71 & 66 & 75 & 59 & 81 & 47 & 80 & 33 & 78 \\
\hline $\begin{array}{c}\text { Gudmundsson } \\
\text { Clayton [1992] } \\
\text { degree 1-10 } \\
\end{array}$ & 88 & 70 & 64 & 73 & 57 & 77 & 46 & 75 & 34 & 78 \\
\hline $\begin{array}{c}\text { Hager \& } \\
\text { Clayton WO } \\
\text { [1989] }\end{array}$ & 4.2 & 7.6 & 3.1 & 3.4 & 11 & 4.7 & 24 & 5.7 & 31 & 6.8 \\
\hline $\begin{array}{c}\text { Hager \& } \\
\text { Richards WL } \\
\text { [1989] }\end{array}$ & 2.2 & 3.5 & 4.8 & 6.7 & 5.7 & 5.5 & 8.1 & 4.2 & 8.4 & 2.1 \\
\hline
\end{tabular}

acting over the area of the CMB would result in a torque of this magnitude. The maximum amplitudes of the contributions to the equivalent torque integral plotted in Figure 4 are more than a factor of 100 greater than this average value.

The contributions to the torque integral for the two flow models at different epochs are similar, but there are discernable differences. For example, beneath southern Africa, the largest negative torque integral contribution for $1960-1970$ has decreased in magnitude by 1980 - 1970, but the magnitude of the negative contribution south of India has increased. During the same interval, the contribution beneath Alaska changes sign, while the maximum over Manchuria splits into separated highs. Although these two flow models, and the patterns of torque contributions computed from them, are similar, the integrated effect of their small differences leads to calculated changes of LOD that differ by almost $2 \mathrm{msec} /$ decade, comparable to the total predictions at the two epochs. Interestingly, the difference in LOD change predicted for these two models is comparable to the difference in LOD change observed for these two decades. But different flow models for the same epochs produce changes in predicted LOD comparable in magnitude, but opposite in sign. For this reason, we cannot assign very high significance to detailed LOD predictions. Indeed, it would probably be possible to construct an acceptable core flow model that exerts no net torque on the mantle [Bloxham, 1991; Voorhies, 1991b]. However, the order of magnitude supports the hypothesis that decadal fluctuations in LOD are largely effected by the topographic torque, even though the timeaveraged torque is, of course, equal to zero. [Hide, 1969].

The effect of spherical harmonic degree truncation in the topography fields can be investigated by comparing the contributions in the torque integral and changes in length of day for two representations of the CMB topography model of Gudmundsson and Clayton [1992]. The first representation, shown in Figure 2, is expanded through degree and order 4 . The second carries the expansion through degree and order 10. (Because only 25 parameters were used in their inversion, the amplitudes of the coefficients fall off fairly rapidly with harmonic degree.) As can be seen from Table 1, increasing the maximum degree and order from 4 to 10 has a very small effect on calculated LOD variations. Inspection of maps of the contributions to the torque integral calculated using Voorhies' flow model and these two topographic models (Figure 5) indicates that most of the features are well-represented by the smoother CMB models. The relative amplitudes of the contributions from different geographic regions vary, but the total torque remains almost constant. We take the stability of these estimates to indicate that contributions from higher harmonics (and their associated errors) are probably insignificant, owing to a high degree of cancellation at these scales (see equation (2.4)).

\section{DISCUSSION AND CONCLUSIONS}

The determination of the efficacy of topographic coupling is clearly fraught with practical difficulties. But the results of this paper demonstrate the usefulness of the method employed by providing independent evidence in favor of the hypothesis that topographic coupling can account for the observed decadal changes in LOD if $\tilde{h}$, the rms. value of the $\phi$-dependent portion of $h$, the topographic relief, is typically about $1 \mathrm{~km}$ in amplitude or possibly slightly less.

We emphasize that this value of $\tilde{h}$ depends on the suppositions underlying the method. In particular, the velocity fields as determined from geomagnetic secular variation data (see Section 3) refer 

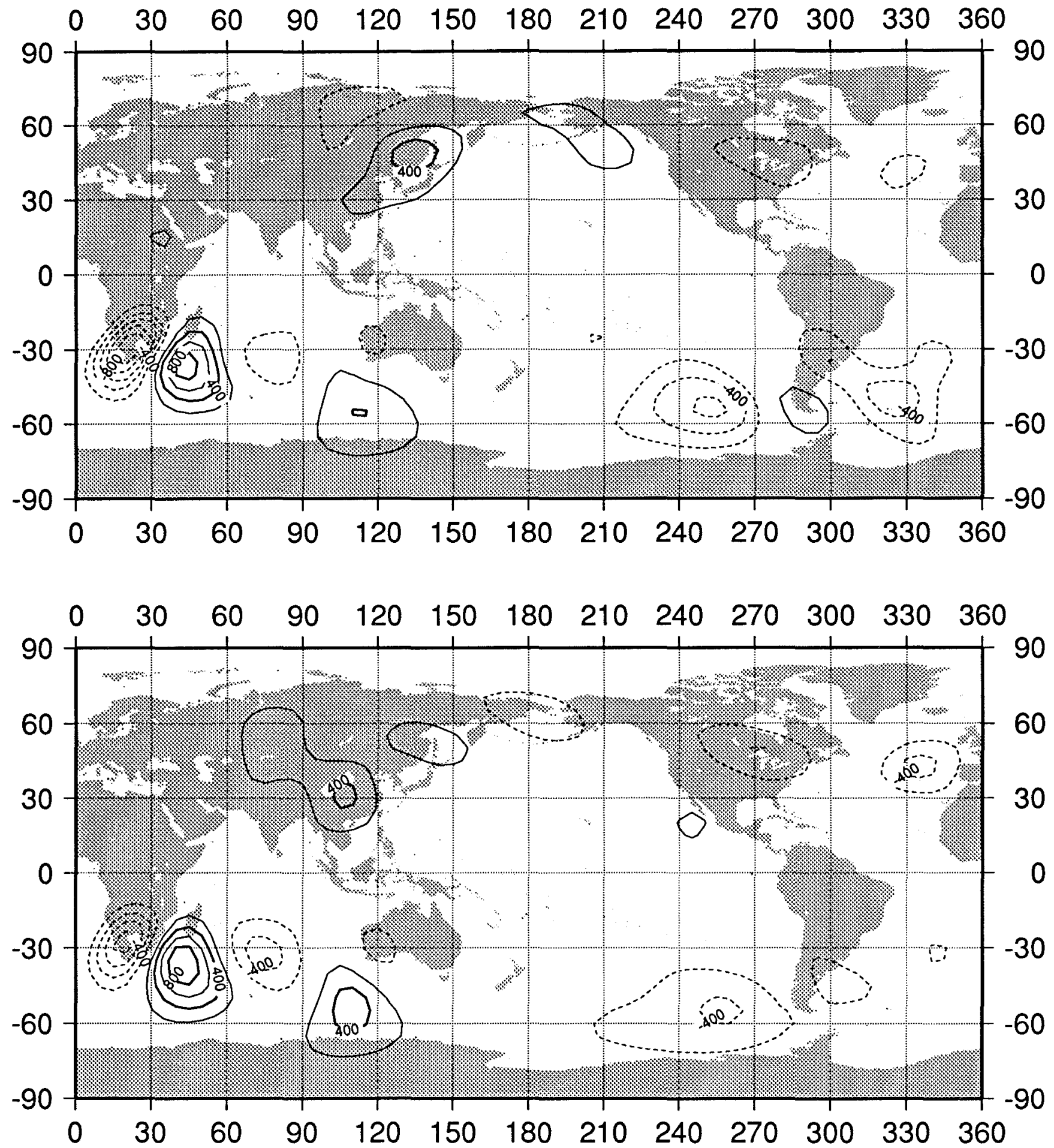

Fig. 4. The contributions to the $L_{3}$ component of torque, in units of $10^{3} \mathrm{~N} / \mathrm{m}$, calculated from the flow models shown in Figure $2 \mathrm{c}$ and $\mathrm{d}$ interacting with the topography shown in the lower panel of Figure 3 . Solid contours indicate positive contributions and dashed contours indicate negative contributions; the zero contour is not shown. The contour interval in both panels is $200 \mathrm{kN} / \mathrm{m}$. The net change in the LOD is related to the integral of these contributions over the area of the CMB. A constant value of $200 \mathrm{kN} / \mathrm{m}$, integrated over the area of the CMB, would give a torque of $0.3 \times 10^{18} \mathrm{Nm}$ and a variation in LOD of $-1.4 \mathrm{msec} / \mathrm{dec}$ ade. 


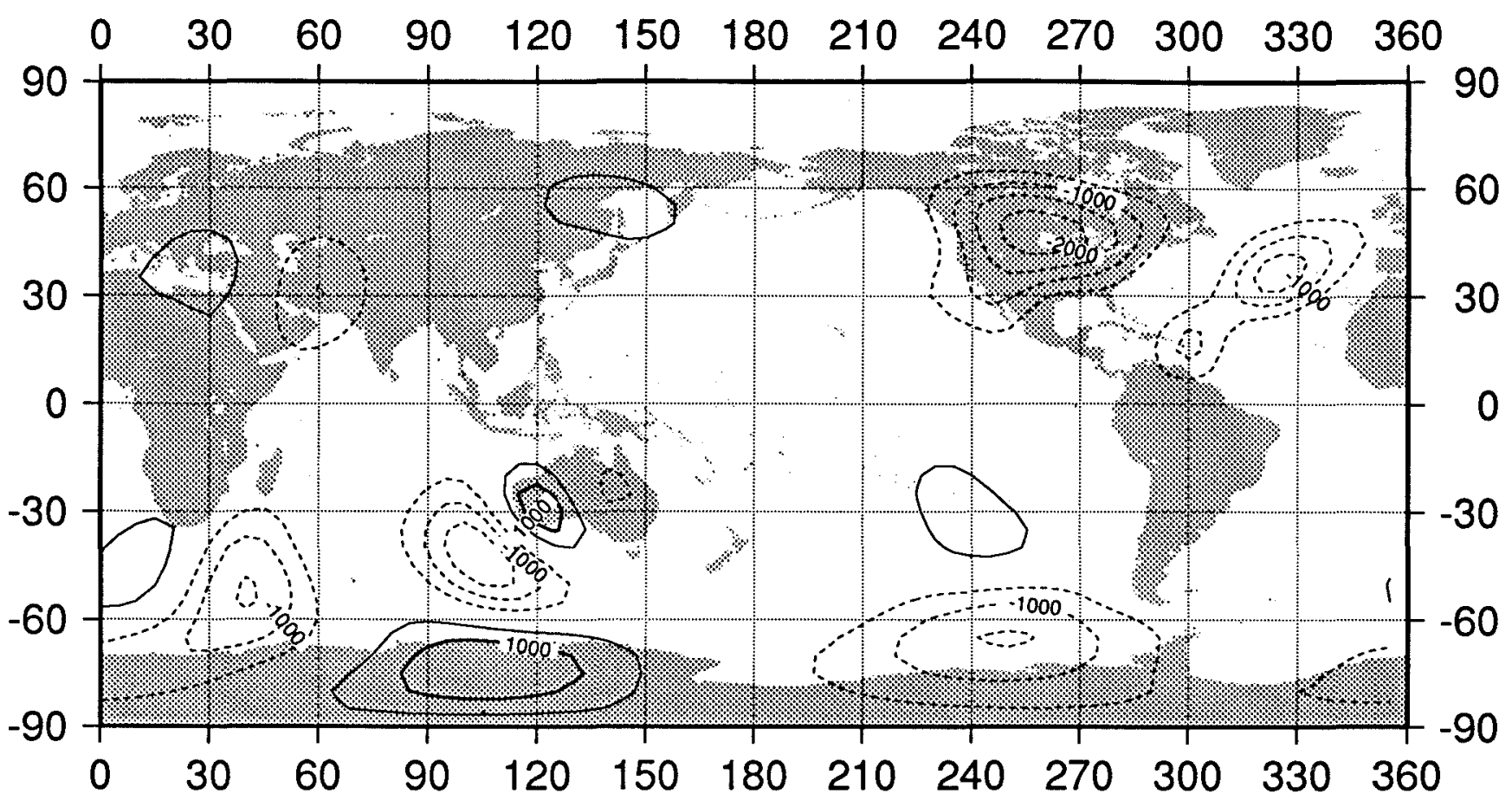

$\begin{array}{lllllllllllll}0 & 30 & 60 & 90 & 120 & 150 & 180 & 210 & 240 & 270 & 300 & 330 & 360\end{array}$

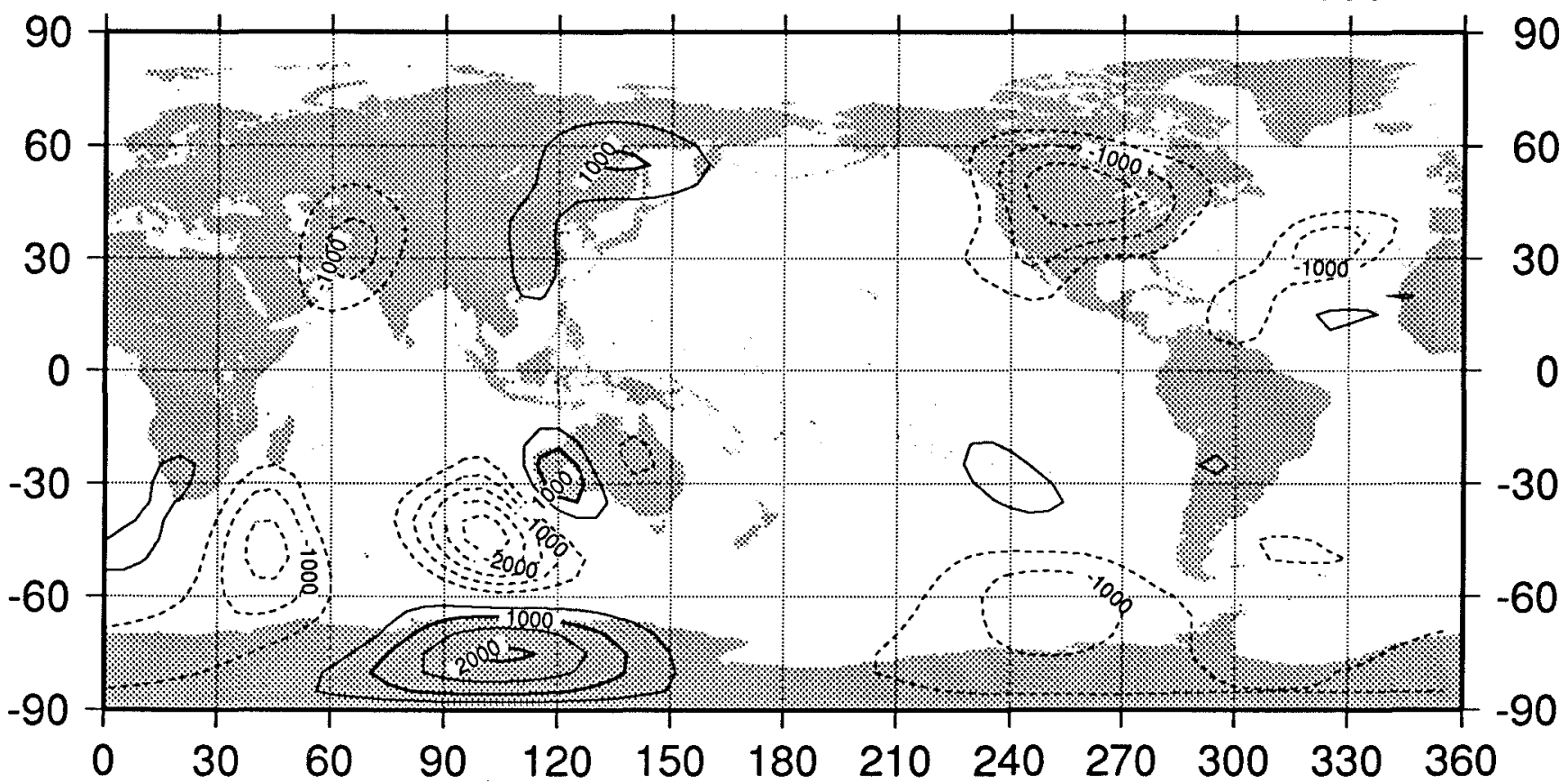

Fig. 5. The contributions to the $L_{3}$ component of torque, in units of $10^{3} \mathrm{~N} / \mathrm{m}$, calculated from the flow model shown in Figure $2 \mathrm{~b}$ interacting with the topography from the model of Gudmundsson and Clayton [1992] for two different degrees of truncation. The upper panel is for topography expanded through degree and order 4 (shown in the upper panel of Figure 3). The lower panel is for the model expanded through degree and order 10. Solid contours indicate positive contributions. The contour interval in both panels is $500 \mathrm{kN} / \mathrm{m}$. The net change in the LOD is related to the integral of these contributions over the area of the CMB. 
to the free stream at the top of the electrically-conducting liquid core. In calculating from these velocity fields the pressure fields acting upon topographic relief of the CMB, it has been assumed that the electrically-conducting core is in contact with the mantle over the whole of the CMB. This is probably a reasonable supposition, but it is interesting to speculate on the possibility that between the highly conducting core and the weakly conducting lower mantle there might exist a thin continuous layer or "pools" of weakly-conducting or insulating liquid which have escaped detection by seismic methods. The actual pressure field at the CMB might then differ from that given by equation (2.4) [Hager and Richards, 1989]. It would have to be determined from considerations of the dynamics of the hypothetical intermediate layer or pools, and the relationship between the two pressure fields might not be simple. If typical pressure gradients at the CMB were weaker than those at the top of the electricallyconducting core, then the value of $\tilde{h}$ needed to produce the necessary torques on the mantle would have to be larger. However, the idea of an intermediate layer is not supported by a parallel study to the present one [R. Hide. and A. Jackson, in preparation], in which decadal polar motion induced by topographic core-mantle coupling is investigated.

As we have seen in Section 4, inferences concerning $\tilde{h}$ can be drawn from direct seismic measurements and also from attempts to interpret the horizontal variations of the Earth's gravitational field in terms of density variations in the mantle maintained by slow convection there. In both cases, the implied value of $\tilde{h}$ depends critically on the assumptions made concerning the properties of the lower mantle. Seismic measurements give values greater than $1 \mathrm{~km}$ unless there are substantial $(\sim 1 \%)$ lateral variations in seismic velocities in the zone just above the $\mathrm{CMB}$. The most likely candidate is the D" layer of $100-300 \mathrm{~km}$ thickness. Consistent with this "scenario" are the mantle convection studies, which give $\tilde{h}$ of about $1 \mathrm{~km}$ where a chemically-distinct or low viscosity layer in the D"region is included, but a significantly greater value otherwise.

So far as future work on topographic core-mantle coupling is concerned, as better geophysical and geodetic data and models become available it will be necessary to repeat, refine, and extend the calculations and comparisons made in the present paper along the obvious lines already indicated. The results will have important implications not only for the nature of the stresses responsible for torques at the $\mathrm{CMB}$ and the structure of the lower mantle and $\mathrm{CMB}$ topography, but also, indirectly, for the magnetohydrodynamics of the core and the nature of the geodynamo. Dynamo "models" can be classified in terms of two characteristic features, namely (a) the average strength of the toroidal magnetic field in the core (which for "strong field" dynamos is typically much greater than that of the poloidal field, whereas for "weak field" dynamos the two field types are comparable in strength), and (b) whether or not dynamo action extends throughout most of the volume of the core or is confined to the upper reaches. Should more refined calculations either weaken the case for significant topographic coupling, or consistently indicate an excessive topographic couple, this would constitute evidence in favor of electromagnetic torques at the $\mathrm{CMB}$ produced by dynamo action concentrated just below the CMB.
Acknowledgements. We thank Don Anderson, Jean Dickey, Olafur Gudmundsson, David Stevenson, and Charles Yoder for helpful comments on various aspects of this work. We also thank Dr. Dickey and other members of the Space Geodesy Science and Applications Group of the Jet Propulsion Laboratory for facilitating this collaborative sudy. Jeremy Bloxham kindly provided coefficients for his core flow model. Partial support was provided by NASA grants NAG5-819 and NAG5-315 to RWC and BHH.

\section{REFERENCES}

Aldridge, K.D. (ed), Core-mantle interactions, Surveys in Geophys., $11,329-353,1990$.

Anderson, D.L. Theory of the Earth, Oxford, Blackwell Scientific Publications, 1989.

Anufriev, A.P., and S.L. Braginsky, Influences of irregularities in the boundary of the Earth's core on fluid velocity and magnetic field, Geomagn. Aeron., 17, 492-496, 1977.

Babcock, A., and G. Wilkins, (Eds.) The Earth's Rotation and Reference Frames, Reidel, Dordrecht, 1988.

Backus, G.E., Kinematics of secular variation in a perfectly conducting core, Philos. Trans. Roy. Soc., A263, 239-263, 1968.

Backus, G.E., and J-L Le Mouël, The region of the core-mantle boundary where the geostrophic velocity field can be determined from frozen flux data, Geophys. J. Roy. Astron. Soc., 85, 617-628, 1986.

Backus, G.E., and J-L Le Mouël, The region of the core-mantle boundary where the geostrophic velocity field can be determined from frozen flux data, addendum, Geophys. J. Roy. Astron. Soc., $88,321-322,1987$.

Benton, E.R., Magnetic probing of planetary interiors, Phys. Earth Planet. Int., 20, 111-118, 1979.

Benton, E.R., A simple method for determining the vertical growth rate of vertical motion at the top of Earth's outer core, Phys. Earth Planet. Int., 24, 242-244, 1981a.

Benton, E.R., Inviscid, frozen-flux velocity components at the top of Earth's core from magnetic observations at earth's surface: Part 1. A new methodology, Geophys. Astophys. Fluid Dyn., 18, 154-174, $1981 \mathrm{~b}$.

Benton E. R., and M. A. Celaya, The simplest unsteady surface flow of a frozen flux core that exactly fits a geomagnetic field model, Geophys. Res. Lett., 18, 577-580, 1991.

Bloxham, J., The dynamical regime of fluid flow at the core surface, Geophys. Res. Lett., 15, 585-588, 1988.

Bloxham, J., Length-of-day variations, topographic core-mantle coupling, and the steady and time-dependent components of core flow (abstract), Eos, Trans. AGU, 72, 451, 1991.

Bloxham, J., Simple models of fluid flow at the core surface derived from geomagnetic field models, Geophys. J. Int., 99, 173-182, 1989.

Bloxham, J., and A. Jackson, Fluid flow near the surface of the Earth's core, Rev. Geophys., 29, 97-120, 1991.

Bullard, E.C., C. Freedman, H. Gellman, and J. Nixon, The westward drift of the Earth's magnetic field, Phil. Trans. Roy. Soc., A243, 6792,1950

Bullen, K.E., Introduction to the Theory of Seismology, Cambridge University Press, Cambridge, 1963.

Cazenave, A., editor, Earth Rotation; Solved and Unsolved Problems, NATO Advanced Institute Series C; Mathematical and Physical Sciences, Boston, D. Reidel, 1986.

Courtillot, V., and J-L Le Mouël, Time variations of the Earth's magnetic field: From daily to secular, Ann. Rev. Earth Planet. Sci., 16, 389-476, 1988.

Dickey, J.O., T.M. Eubanks, and R. Hide, Interannual and decade flunctuations in the Earth's rotation, in Variations in the Earth's Rotation, Geophysical Monograph Series, edited by D.C. McCarthy, pp. 157-162, AGU, Washington, DC, 1990.

Doombos, D.J., The effect of a rough core-mantle boundary found only on PKKP, Phys. Earth Planet. Int., 21, 351-358, 1980. 
Eltayeb, I.A., and M.H.A. Hassan, On the effects of a bumpy coremantle interface, Phys. Earth Planet. Int., 19, 239-254, 1979.

Gire, C., and J-L Le Mouêi, Tangentially-geostrophic flow at the coremantle boundary compatible with observed geomagnetic secular variation: The large-scale component of the flow, Phys. Earth Planet. Int., 59, 259-287, 1990.

Gire, C., J-L. Le Mouël, and T. Madden, Motions at the core surface derived from SV data, Geophys. J. R. Astr. Soc., 84, 1-29, 1986.

Gubbins, D., Finding core motions from magnetic observations, Phil. Trans. Roy. Soc., A306, 247-254, 1982.

Gudmundsson, O., and R.W. Clayton, A 2-D synthetic study of global traveltime tomography, Geophys. J. Int., 106, 53-68, 1991.

Gudmundsson, O., and R.W. Clayton, Some problems in mapping core-mantle boundary structure, J. Geophys. Res., in press, 1992.

Gudmundsson, O., J.H. Davies, and R.W. Clayton, Stochastic analysis of global travel time data: Mantle heterogeneity and random errors in the ISC data, Geophys. J. Int., 102, 25-43, 1990.

Gwinn, C.R., T.A. Herring, and I.I. Shapiro, Geodesy by radio interferometry: Studies of the forced nutations of the Earth, 2. Interpretation, J. Geophys. Res., 91, 4755-4765, 1986.

Haddon, R. A. W., Evidence of inhomogeneities near the core-mantle boundary, Phil. Trans. Roy. Soc., A306, 61-70, 1982.

Hager, B. H., and R. W. Clayton, Constraints on the structure of mantle convection using seismic observations, flow models, and the geoid, in Mantle Convection, edited by W. R. Peltier, pp. 657-763, Gordon and Breach, New York, 1989.

Hager, B.H., R. W. Clayton, M. A. Richards, R. P. Comer, and A. M. Dziewonski, Lower mantle heterogeneity, dynamic topography and the geoid, Nature, 313, 541-545, 1985.

Hager, B.H., and M.A. Richards, Long wavelength variations in the Earth's geoid: Physical models and dynamical implications, Philos. Trans. Roy. Soc., A328, 309-327, 1989.

Herring, T.A., B. A. Buffett, P. M. Mathews, and I. I. Shapiro, Forced motions of the Earth: Influence of inner core dynamics: 3 . Very long interferometry data analysis, J. Geophys. Res., 96, 8259-8273, 1991.

Herring, T.A., C.R. Gwinn, and I.I. Shapiro, Geodesy by radio interferometry: Corrections to the IAU 1980 nutation series, Proc. Inter. Conf. Earth Rotation and Terrestrial Reference Frame, 1, 307-328, Ohio State University, Columbus, 1985.

Herring, T.A., C.R. Gwinn, and I.I. Shapiro, Geodesy by radio interferometry: Studies of the forced nutations of the Earth 1. Data analysis, J. Geophys. Res., 91, 4745-4754, 1986.

Hide, R., Interaction between the Earth's liquid core and solid mantle, Nature, 222, 1055-1056, 1969.

Hide, R., On the Earth's core-mantle interface, Quart. J. Roy. Meteorol. Soc., 96, 579-590, 1970.

Hide, R., Towards a theory of irregular variations in the length of the day and core-mantle coupling, Phil. Trans. Roy. Soc., A284, 547554, 1977.

Hide, R., Presidential address: The Earth's differential rotation, Quart. J. Roy. Astron. Soc., 278, 3-14, 1986.

Hide, R., Fluctuations in the Earth's rotation and the topography of the core-mantle interface, Phil. Trans. Roy. Soc., A328, 351-363, 1989.

Hide, R., and J.O. Dickey, Earth's variable rotation, Science, 253, 629$637,1991$.

Hide, R., and K.I. Horai, On the topography of the core-mantle interface, Phys. Earth Planet. Int., 1, 305-308, 1968.

Hills, R.G., Convection in the Earth's mantle due to viscous stress at the core-mantle interface and due to large scale buoyancy. Ph.D. thesis, New Mexico State University, Las Cruces, 1979.

Hinderer, J., D. Jault, and J-L Le Mouël, Core-mantle topographic torque: A spherical harmonic approach and implications for the excitation of the Earth's rotation by core motions, Phys. Earth Planet. Int., 59, 329-341, 1990.

Jacobs, J.A., (Ed.) The Earth's Core, 2nd ed., Academic Press Ltd., New York, $1987 a$.
Jacobs, J.A. Geomagnetism. 2 vols. Academic Press Ltd., New York, $1987 \mathrm{~b}$.

Jault, D., and J-L Le Mouël, The topographic torque associated with tangentially geostrophic motion at the core surface and inferences on the flow inside the core, Geophys. Astrophys. Fluid Dyn., 48, 273-296, 1989.

Jault, D., and J-L Le Mouël, Core-mantle boundary shape: Constraints inferred form the pressure torque acting between the core and mantle, Geophys. J. Int., 101, 233-241, 1990.

Jault, D., and J-L Le Mouël, Exchange of angular momentum between the core and mantle, J. Geomag. Geoelect., 43, 111-129, 1991.

Jault, D., C. Gire, and J-L Le Mouël, Westward drift, core motions and exchange of angular momentum between core and mantle, Nature, $333,353-356,1988$.

Jeanloz, R., The nature of the Earth's core, Ann. Rev. Earth Planet. Sci., 18, 257-386, 1990.

Kinoshita, H., and J. Souchay, The theory of the nutation for the rigid Earth model of second order, Celestial Mechanics, 48, 187-265, 1990.

Knittle, E., and R. Jeanloz, Earth's core-mantle boundary: Results of experiments at high pressures and temperatures, Science, 251, 1438-1443, 1991.

Lambeck, K., The Earth's Variable Rotation, Cambridge University Press, London and New York, 1980.

Lambeck, K., Geophysical Geodesy: The Slow Deformation of the Earth, Oxford, Clarendon Press, 1988.

Le Mouël, J-L, Outer-core geostrophic flow and secular variation of the main geomagnetic field, Nature, 311, 734-735, 1984.

Lloyd, D., and D. Gubbins, Toroidal fluid motion at the top of the Earth's core, Geophys. J. Int., 100, 455-467, 1990.

Loper, D.E., The nature and consequences of thermal interactions twixt core and mantle, J. Geomag. Geoelect., 43, 79-91, 1991.

Melchior, P. The Physics of the Earth's Core. Pergamon Press, Oxford , 1986.

Moffatt, H.K., Magnetic Field Generation by Fluid Motion, Cambridge University Press, Cambridge, 1978a.

Moffatt, H.K., Topographic coupling at the core-mantle interface, Geophys. Astrophys. Fluid Dyn., 9, 279-288, 1978 b.

Morelli, A., and A.M. Dziewonski, Topography of the core-mantle boundary and lateral homogeneity of the liquid core, Nature, 325 , 678-683, 1987.

Moritz, H., and I.I. Mueller (Eds.), Earth Rotation: Theory and Observation, The Ungar Publishing Co., New York, 1987.

Morrison, L.V., Redetermination of the decade fluctuations in the rotation of the Earth in the period 1861-1978, Geophys. J. Roy. Astron. Soc., 38, 349-360, 1979.

Munk, W.H., and G.J.F. MacDonald, The Rotation of the Earth, Cambridge University Press, Cambridge, 1960.

Paulus, M., and M. Stix, Electromagnetic core-mantle coupling: The Fourier method for solving the induction equation, Geophys. Astrophys. Fluid Dyn., 47, 237-249, 1989.

Reid, M.J., and J.M. Moran, (Eds.) The Impact of VLBI on Astrophysics and Geophysics, Kluwer Academic Publishers, Dordrecht, 1988.

Roberts, P. H., Electromagnetic core-mantle coupling, J. Geomag. Geoelect., 24, 231-259, 1972.

Roberts, P.H., and S. Scott, On analysis of secular variation: 1 A hydromagnetic constraint, J. Geomag. Geoelect., 17, 137-151, 1965.

Rochester, M.G., Causes of fluctuations in the Earth's rotation, Phil. Trans. Roy. Soc., A313, 95-105, 1984.

Spieth, M.A. R. Hide, R. W. Clayton, B. H. Hager, and C. V. Voorhies, Topographic coupling of the core and mantle and changes in the length of the day (abstract), Eos Trans. AGU, 67, 908, 1986.

Stoyko, N., Sur les variations de champ magnetique et la rotation de la Terre, C.R. Hebd. Seanc. Acad. Sci. Paris, 233, 80-82, 1951.

Vestine, E.H., On variations of the geomagnetic field, fluid motions, 
and the rate of the Earth's rotation, Proc. Nat. Acad. Sci., 38, 1030 1038, 1952.

Voorhies, C. V., Steady flows at the top of Earth's core derived from geomagnetic field models, J. Geophys. Res., 91, 12, 444-12, 466, 1986 .

Voorhies, C.V., Steady surficial core motions: an altemate method. Geophys. Res. Lett., 13, 1537-1540, 1986 b.

Voorhies, C.V., The time-varying geomagnetic field, Rev. Geophys., $25,929-938,1987$.

Voorhies, C.V., Probing surface core motions with DGRF models (abstract), Eos Trans. AGU, 69, 336, 1988.

Voorhies, C.V., Coupling an inviscid core to an electrically-insulating mantle, J. Geomag. Geoelect., 43, 131-156, 1991 a.

Voorhies, C.V., On the joint inversion of geophysical data for models of the coupled core-mantle system, NASA Tech Memo 104536, 24pp., $1991 \mathrm{~b}$.

Voorhies, C. V., Implications of decade fluctuations in the length of the day for geomagnetic estimates of core surface flow and geodynamo experiments, in Geophysical Monographs, edited by J-L. Le Mouël, submitted, 1992.

Voorhies, C.V., and G.E. Backus, Steady flows at the top of the core from geomagnetic field models: the steady motions theorem. Geophys. Astrophys. Fluid Dyn., 32, 163-173, 1985.

Wahr, J. M., The Earth's rotation, Ann. Rev. Earth Planet. Sci., 16, 231-249, 1988.

Whaler, K.A., Geomagnetic evidence for fluid upwelling at the core- mantle boundary, Geophys. Journ. Roy. Astron. Soc., 86, 563-588, 1986.

Whaler, K.A., A steady velocity field at the top of the Earth's core in the frozen-flux approximation - errata and further comments, Geophys. J. Int., I02, 507-509, 1990.

Whaler, K.A., Properties of steady flows at the core-mantle boundary in the frozen-flux approximation, Phys. Earth Planet. Int., 68, 144$155,1991$.

Whaler, K.A., and S.O. Clarke, A steady velocity field at the top of the Earth's core in the frozen-flux approximation. Geophys. I. Int., 94, 143-155, 1988.

R. W. Clayton, Seismological Laboratory, California Institute of Technology, Pasadena, CA 91125. U. S. A.

B. H. Hager, Department of Earth, Atmospheric and Planetary Sciences, Massachusetts Institute of Technology, Cambridge, MA 02139, U. S. A.

R. Hide, Robert Hooke Institute, The Observatory, Clarendon Laboratory, Parks Road, Oxford OX1 3PU, England, U. K.

M. A. Spieth, Jet Propulsion Laboratory, Califomia Institute of Technology, Pasadena, CA 91109, U. S. A.

C. V. Voorhies, Geodynamics Branch Code 921, Goddard Space Flight Center, Greenbelt, MD 20771, U. S. A. 